\title{
ON THE LOCATION OF THE ZEROS OF THE DERIVATIVES OF A POLYNOMIAL SYMMETRIC IN THE ORIGIN
}

\author{
J. L. WALSH
}

If the zeros of a polynomial $p(z)$ when plotted in the $z$-plane are symmetric in $0: z=0$, the zeros of the derivative $p^{\prime}(z)$ of $p(z)$ can profitably be studied by transforming onto the $w$-plane, with $w=z^{2}$, and applying known theorems there. ${ }^{1}$ It is the purpose of the present note to carry that study somewhat farther than has been previously done, in particular to consider the higher derivatives of $p(z)$.

Under the transformation $w=u+i v=z^{2}=(x+i y)^{2}$, an arbitrary line $A u+B v+C=0$ in the w-plane corresponds to an equilateral hyperbola $A\left(x^{2}-y^{2}\right)+2 B x y+C=0$ in the $z$-plane with center 0 or to two perpendicular lines intersecting at 0 . A half-plane in the wplane for which $w=0$ is an interior or exterior point corresponds in the z-plane respectively to the exterior or interior of an equilateral hyperbola whose center is 0 ; a half-plane for which $w=0$ is a boundary point corresponds to a double sector with vertex $z=0$ and angle $\pi / 2$. A point $z$ is considered to be exterior or interior to a hyperbola according as the curve at its nearest point is convex or concave toward $z$.

We write the given polynomial in the form

$$
p(z)=z^{l} \prod_{j=1}^{q}\left(z^{2}-\alpha_{j}^{2}\right), \quad \quad \alpha_{j} \neq 0,
$$

and in the $w$-plane study the polynomials $\left(w=z^{2}\right)$

$$
P(w)=P\left(z^{2}\right)=[p(z)]^{2}, \quad P^{\prime}(w)=p(z) \cdot p^{\prime}(z) / z .
$$

Each zero of $P(w)$ corresponds to a zero of $p(z)$ and reciprocally; each zero of $P^{\prime}(w)$ corresponds to a zero of $p(z)$ or $p^{\prime}(z)$ and reciprocally except that $z=0$ is a zero of $p^{\prime}(z)$ unless $z=0$ is a simple zero of $p(z)$.

We have (loc. cit.) by Lucas' Theorem

THEOREM 1. If the zeros of $p(z)$ are symmetric in 0 and lie in the closed exterior of an equilateral hyperbola with center 0 or in the closed exterior of a double sector with vertex 0 and angle $\pi / 2$, then the zeros of $p^{\prime}(z)$ lie also in that closed exterior.

If the zeros of $p(z)$ are symmetric in 0 and lie in the closed interior of an equilateral hyperbola with center 0 , then the zeros of $p^{\prime}(z)$ also lie in that closed interior except for a simple zero at 0.

Received by the editors December 4, 1947.

${ }^{1}$ Walsh, Mathematica vol. 8 (1933) pp. 185-190. 
To the polynomial $P(w)$ we now apply the theorem:2

Let $P(w)$ be a polynomial in w of degree $n$ with an l-fold $(l>0)$ zero at $w=0$ and all the remaining zeros of $P(w)$ in the closed half-plane $\Pi$ not containing $w=0$. Then except for a zero at $w=0$ of multiplicity $l-1$, all zeros of $P^{\prime}(w)$ lie in the closed half-plane obtained by shrinking $\Pi$ toward the origin in the ratio $n: l$.

The degrees of $p(z)$ and $P(w)$ defined by (1) and (2) are equal, as are the multiplicities of their zeros $z=0$ and $w=0$. Under the transformation $w=z^{2}$, the line $u=a$ corresponds to the hyperbola $x^{2}-y^{2}$ $=a$, and the line $u=l a / n$ corresponds to $x^{2}-y^{2}=l a / n$, so we have

THEOREM 2. Let $p(z)$ be a polynomial of degree $n$ whose zeros are symmetric in the origin 0 , let 0 be an l-fold zero $(l>0)$, and let all the other zeros lie in the closed interior of an equilateral hyperbola $H$ with center 0. Then except for an (l-1)-fold zero at 0 , all zeros of $p^{\prime}(z)$ lie in the closed interior of the hyperbola obtained by shrinking $H$ toward 0 in the ratio $(n: l)^{1 / 2}$.

We turn now to the higher derivatives of the polynomial $p(z)$. Under the conditions of the first part of Theorem 1, the zeros of every derivative of $p(z)$ not vanishing identically lie in the given closed region.

Suppose, however, the zeros of the polynomial $p(z)$ of degree $n$ lie in the closed interior of an equilateral hyperbola $H$ whose center is 0 . The polynomial $p^{\prime}(z)$ has a simple zero at 0 , and by Theorem 1 the remaining zeros of $p^{\prime}(z)$ lie in the closed interior of $H$. By Theorem 2, all zeros of $p^{\prime \prime}(z)$ lie in the closed interior of the hyperbola obtained by shrinking $H$ toward 0 in the ratio $[(n-1): 1]^{1 / 2}$. The higher derivatives of $p(z)$ not vanishing identically have alternately a simple zero at 0 and no zero at 0 . Continued application of Theorems 1 and 2, in alternation, then yields further hyperbolas which respectively contain the zeros other than 0 of the $k$ th derivative of $p(z)$. However, except in the cases $k=1$, and $k=2$ with $n \geqq 4$, these hyperbolas are not the most favorable that can be obtained; a zero of $p^{\prime}(z)$ in Theorem 2 cannot lie on the new hyperbola unless all zeros of $p(z)$ lie on $H$ in two points symmetric in 0 . We proceed to prove the following generalization of Theorem 2, the principal result of the present note:

THEOREM 3. Let $p(z)$ be a polynomial of degree $n$ whose zeros are symmetric in 0 , let 0 be an l-fold zero, and let the remaining zeros lie in the closed interior of a hyperbola $H$ whose center is 0 . Let $p_{0}(z)$ be the polynomial of degree $n$ whose zeros are symmetric in 0 , which has an l-fold

${ }^{2}$ Walsh, Trans. Amer. Math. Soc. vol. 22 (1921) pp. 101-116; p. 115. 
zero at 0 , and whose remaining zeros lie at the vertices of $H$. Let $z_{k}$, $1 \leqq k \leqq n-2$, be one of the zeros of $p_{0}^{(k)}(z)$ of smallest positive modulus, and let $H_{k}$ be the equilateral hyperbola with center 0 which has $z_{k}$ as a vertex. Then all zeros of $p^{(k)}(z)$ other than 0 lie in the closed interior of $H_{k}$.

Of course 0 need not be a zero of $p^{(k)}(z)$; whether or not 0 is such a zero depends on $l, n$, and $k$. To prove Theorem 3 we need a more powerful result than that used in proving Theorem 2 :

Let $Q(w)$ be a polynomial in $w$ of degree $q$, and let the numbers $A_{j}$ be constants. If the locus of the zeros of $Q(w)$ is a closed half-plane $\Pi$, then the locus of the zeros of the polynomial

$$
A_{0} w^{r} Q(w)+A_{1} w^{r+1} Q^{\prime}(w)+\cdots+A_{q} w^{r+q} Q^{(q)}(w)
$$

is also a number of half-planes, identical with the locus of the zeros of the polynomial (3) with $Q(w)$ replaced by $(w-\alpha)^{q}$ :

$$
\begin{aligned}
A_{0} w^{r}(w-\alpha)^{q}+q A_{1} w^{r+1}(w-\alpha)^{q-1} & +\cdots \\
& +q(q-1) \cdot \cdots \cdot 1 A_{q} w^{r+q}
\end{aligned}
$$

when the locus of $\alpha$ is $\Pi$.

The theorem just quoted is essentially a special case of a more general theorem ${ }^{3}$ concerning a linear combination of products of derivatives of two polynomials. If polynomial (4) is written as the product of $w^{r+q}$ and a polynomial in $W=(w-\alpha) / w$ whose zeros are $W=W_{1}, W_{2}, \cdots, W_{q}$, it is seen that the common locus of the zeros of (3) and (4) consists of the origin $w=0$ (provided $w=0$ is a zero of (4)) plus a number of half-planes, loci of the points $w=\alpha /\left(1-W_{j}\right)$, $j=1,2, \cdots, q$, when the locus of $\alpha$ is $\Pi$; if $I I$ does not contain the point $w=0$, a zero $W_{j}=1$ is to be ignored; if $\Pi$ contains the point $w=0$ and if $W_{j}=1$ is a zero of (4), the entire plane is to be considered the locus of the zeros of (3) and (4). If $\Pi$ does not contain 0 , and if for real positive $\alpha$ all zeros of (4) lie in the interval $0 \leqq w \leqq \alpha$, then the locus of the zeros of (4) is the origin (if $w=0$ is a zero of (4)) plus a half-plane not containing 0 bounded by a line parallel to the boundary of $\Pi$, traced by the zero of (4) nearest to but different from 0 when $\alpha$ traces the boundary of $\Pi$.

In Theorem 3 we write

$$
\begin{aligned}
& p(z)=z^{l} p_{1}\left(z^{2}\right), \\
& p_{1}(0) \neq 0, \\
& p^{\prime}(z)=l z^{l-1} p_{1}\left(z^{2}\right)+2 z^{l+1} p_{1}^{\prime}\left(z^{2}\right),
\end{aligned}
$$

${ }^{3}$ Walsh, Trans. Amer. Math. Soc. vol. 24 (1922) pp. 163-180; Theorem 9 of that paper applies to two circular regions, here specialized to a single point and a halfplane respectively. 


$$
\begin{gathered}
p^{\prime \prime}(z)=l(l-1) z^{l-2} p_{1}\left(z^{2}\right)+(4 l+2) z^{l} p_{1}^{\prime}\left(z^{2}\right)+4 z^{l+2} p_{1}^{\prime \prime}\left(z^{2}\right) \\
\ldots \ldots \ldots \ldots \ldots \ldots \ldots \ldots \ldots \ldots \ldots \ldots \ldots \ldots \ldots \ldots \ldots \ldots
\end{gathered}
$$

Except perhaps for a factor $z$, these derivatives are precisely of the form (3), with $w=z^{2}$. If we have $p_{0}(z)=z^{l}\left(z^{2}-\beta^{2}\right)^{(n-l) / 2}$, the zeros of $p_{0}^{(k)}(z)$ lie on the line segment joining $\beta$ and $-\beta$, and the corresponding zeros in the $w$-plane lie on the segment joining 0 and $\beta^{2}$; the images in the $w$-plane of the zeros of $p_{1}(z)$ lie in a half-plane $\Pi$ not containing $w=0$; thus by the theorem quoted the images in the $w$-plane of the zeros of $p^{(k)}(z)$ other than $z=0$ lie in the half-plane whose boundary is parallel to that of $\Pi$, which contains $\Pi$ in its interior, and whose boundary passes through the image of the zero of $p_{0}^{(k)}(z)$ nearest to but distinct from 0 . Theorem 3 follows.

When as in Theorem 1 we study the zeros of a polynomial $p(z)$ that are symmetric in 0 , equilateral hyperbolas with center 0 are both (1) the lines of force in the z-plane in the Gaussian field due to two particles symmetric in 0 and (2) the images in the z-plane of the straight lines in the $w$-plane under the transformation $w=z^{2}$. Indeed, the lines of force due to particles at $z=\alpha$ and $z=-\alpha$ are loci arg $\left(z^{2}-\alpha^{2}\right)=$ const., arcs of equilateral hyperbolas with center 0 ; halflines in the $w$-plane can be written arg $\left(w-w_{0}\right)=$ const., and their images in the $z$-plane are loci arg $\left(z^{2}-w_{0}\right)=$ const.

A set of points in the $z$-plane is said to have $m$-fold symmetry about 0 if the set is unchanged by a rotation about 0 through an angle of $2 \pi / m$. A polynomial $p(z)$ whose zeros possess $m$-fold symmetry about 0 is readily studied by means of the transformation $w=z^{m}$. Under this transformation a straight line in the $w$-plane not through $w=0$ corresponds to a curve which may be called an $m$-hyperbola with center 0 . A closed half-plane in the $w$-plane containing 0 in its interior corresponds to the closed exterior of an $m$-hyperbola, and a closed halfplane not containing 0 corresponds to the closed interior of an $m$ hyperbola. A line through $w=0$ corresponds to $m$ equally spaced lines through $z=0$, and a half-plane in the $w$-plane bounded by a line through $w=0$ corresponds to $m$ equally spaced sectors in the $z$-plane, each of angle $\pi / m$. The function $\left[p\left(w^{1 / m}\right)\right]^{m}$ is a polynomial in $w$ which is readily studied in the w-plane, and yields results on the zeros of $p^{\prime}(z)$ in the $z$-plane. Theorem 3 extends directly to a polynomial $p(z)$ whose zeros possess $m$-fold symmetry in 0 . The $m$-hyperbolas with center 0 are not merely the images in the z-plane of the straight lines in the w-plane, but also the lines of force in the $z$-plane due to $m$ particles $m$-fold symmetric in $z=0$.

HARVARD UNIVERSITY 